\title{
Condición clínica y niveles de colinesterasa de trabajadores informales dedicados a la fumigación con plaguicidas
}

\author{
Clinical condition and colinesterase levels of informal workers dedicated to \\ fumigation with pesticides
}

José Moisés Luna Rondón', Mónica Isabel Hanna Lavalle', Concepción Elena Amador ${ }^{1}$

\section{Resumen}

Introducción. Las prolongadas exposiciones a diversos plaguicidas pueden alterar la salud de los individuos mediante alteración de la funcionalidad de la enzima colinestera. Objetivo. Determinar los síntomas clínicos por efectos probables de inhibición de las enzimas colinesterasas en un grupo de fumigadores del sector informal de la economía. Materiales y métodos. Diseño analítico, prospectivo. A cada uno de los 256 fumigadores sin otras condiciones susceptibles de afectar la enzima, se le tomó semestralmente muestra de $10 \mathrm{~mL}$ de sangre venosa periférica; hasta completar 4 mediciones. Cada muestra de sangre fue repartida en dos tubos heparinizados, preservados en frío $\left(2-8^{\circ} \mathrm{C}\right)$ hasta 10 horas máximo. El rango de normalidad utilizado como referencia fueron las cifras encontradas para Colombia por Carmona-Fonseca en adultos no expuestos utilizando la técnica $\mathrm{EQM}^{\oplus}$, la misma que ha sido utilizada en este estudio. Resultados. La edad de inicio en la tarea de fumigación fue de 14 a 16 años (73\%), 100\% se mantuvo laboralmente activos, en contacto laboral con insecticidas órgano-fosforados y diversos compuestos activos herbicidas durante tiempo de exposición entre 8 y 28 años (67\%). El promedio de las cuatro mediciones de colinesterasa eritrocitaria en fumigadores osciló entre 33.8 y 27.6, con descensos progresivos del valor inicial (67\%) en las mediciones sucesivas, existe $37.2 \%$ de sintomáticos para intoxicación crónica. Conclusiones. La exposición continuada a plaguicidas refleja alteraciones de la colinesterasa eritrocitaria entre los fumigadores informales. Discusión. Hallazgos similares con Abou-Hatab en relación con resultados y la edad, entre expuestos sintomáticos se evidencia intoxicación crónica.

Palabras claves: ocupación, plaguicidas, colinesterasa, laboratorio, trabajadores informales, exposición ocupacional.

\footnotetext{
1. Docente-investigador Universidad de Córdoba.

Montería, Córdoba. Colombia.

ORCID JMLR: http://orcid.org/0000-0003-0489-1544

ORCID MIHL: http://orcid.org/0000-0002-0008-3374

ORCID CEA: http://orcid.org/0000-0003-3311-0938
} 


\section{Abstract}

Introduction. Prolonged exposures to various pesticides can alter the health of individuals by altering the functionality of the cholinesterase enzyme. Objective. To determine the clinical symptoms by probable effects of inhibition of the cholinesterase enzymes in a group of fumigators of the informal sector of the economy. Materials and methods. Analytical, prospective design. To each of the 256 fumigators without other conditions susceptible to affect the enzyme, a $10 \mathrm{~mL}$ sample of peripheral venous blood was taken every six months; until completing 4 measurements. Aach blood sample was divided into two heparinized tubes, preserved in cold $\left(2-8^{\circ} \mathrm{C}\right)$ up to 10 hours-maximum. The normality range used as a reference was the figures found for Colombia by Carmona-Fonseca in adults not exposed using the $\mathrm{EQM}^{\bullet}$ technique, the same one that has been used in this study. Results. The age of initiation in the fumigation was 14 to 16 years (73\%), $100 \%$ remained labor-active, in labor contact with organophosphorus insecticides and various herbicidal active compounds during exposure time between 8 and 28 years (67\%). The average of the four measurements of erythrocyte cholinesterase in sprayers ranged between 33.8 and 27.6, with progressive decreases of the initial value (67\%) in the successive measurements; there are $37.2 \%$ of symptomatic for chronic intoxication. Conclusions. The continuous exposure to pesticides reflects alterations in the erythrocyte cholinesterase among the informal fumigators. Discussion. Similar findings with Abou-Hatab in relation to results and age, among symptomatic exposed chronic poisoning are evidenced.

Keywords: occupation, pesticides, cholinesterase, laboratory, informal workers, occupational exposure.

\section{Introducción}

Colombia en sus regulaciones, define la enfermedad laboral como la contraída como resultado de la exposición a factores de riesgo inherentes a la actividad laboral o del medio en el que el trabajador se ha visto obligado a trabajar. De acuerdo con reportes oficiales de las estadísticas Presidenciales Ministerio de Salud y Protección Social - Sistema de Información Gremial de la Federación de Aseguradores Colombianos (Fasecolda), en Colombia un $11.0 \%$ de las enfermedades profesionales que han sido calificadas por actividad económica, corresponden al sector de agricultura, pesca y caza que conglomera una cantidad considera- ble de oficios tanto del sector formal como del informal; y como parte de ello, se han realizado algunos avances en las determinaciones de las frecuencias de intoxicaciones por plaguicidas en Colombia (1-3).

En Córdoba (Colombia), debido al predominio de la economía agropecuaria, cerca del $80 \%$ de la fuerza laboral del territorio se localiza en las áreas rurales, en las que existen familias que derivan su sustento diario de actividades informales como sembradores, plagueros, recolectores, macheteros, fumigadores de cultivos, de bovinos y de otras especies menores. Esta última actividad se realiza en las fincas de grandes y pequeños propietarios con 
el fin de controlar las plagas susceptibles de afectar los rendimientos económicos de su explotación agrícola o ganadera.

En curso de esta actividad en la zona de sabanas de Córdoba se ha censado a 785 personas que ejercen de manera informal y diaria esta labor, por lo que de manera continua se exponen laboralmente a una diversidad de compuestos químicos de uso agrícola y pecuario. Debido a la informalidad en la cual se realiza dicha exposición los fumigadores no tienen acceso a los beneficios educativos y de protección laboral que cubre el sistema de riesgos laborales vigente en Colombia. A causa de lo anterior, los fumigadores que trabajan en los campos agropecuarios a pesar del riesgo de intoxicación aguda y crónica desconocen sus niveles de Colinesteresa Eritrocitaria (CE) y sérica.

El grupo de enzimas orgánicas encargadas de realizar la hidrólisis de la acetilcolina y otros ésteres de la colina durante el proceso bioquímico de metabolización se llaman "colinesterasas" y sus resultados e interpretaciones pueden variar dependiendo del método o la técnica utilizada. Si bien es cierto que los plaguicidas actúan como inhibidores de las colinesterasas; también se reconoce que existen otras condiciones susceptibles de afectar las cifras de colinesterasa, como la condición nutricional, según la cual, la desnutrición produce disminución de la CE y la obesidad la altera, reflejando aumento de la CE. Así mismo, algunos medicamentos tienden a reducir los valores de colinesterasa, condiciones que podrían dar lugar a falsos positivos y también a falsos negativos en entre los sujetos en estudio (4-9).

Todas estas condiciones se reflejan en un amplio rango de fluctuaciones interindividuales e intraindividuales, que se expresan en los rangos de normalidad definidos para la interpretación de resultados. Al respecto y para controlar tal dispersión de los datos, Carmona-Fonseca, realizaron en Colombia un estudio para determinar los valores de referencia nacionales en población de hombres adultos no expuestos, utilizando dos técnicas de medición de CE. En cuanto a las mediciones encontrada con la técnica $\mathrm{EQM}^{\oplus}$, se identificó que las cifras basales normales en individuos sanos, no expuestos a plaguicidas, corresponde a 35,21 U/g de oxihemoglobina expresadas en valores aceptables de colinesterasa eritrocitaria (IC95\%) entre 34,82 a $35,60 \mathrm{U} / \mathrm{mL}$ que fueron los utilizados como referentes en la interpretación de los hallazgos $(10,11)$.

Las investigaciones disponibles en el área de toxicidad que se han realizado en el contexto local son muy puntuales y se relacionan con agentes específicos como intoxicación por mercurio en los cuerpos de aguas de las principales ciénagas. Entre los fumigadores sujetos del estudio se han realizados algunos estudios sobre colinesterasa sérica tras la accidentalidad conducente a intoxicación aguda, pero ninguno en el Departamento de Córdoba y el estudio sobre intoxicaciones agudas atendidas (11-14).

Estos fumigadores debido a su trabajo, están sometidos a diario a pequeños contactos accidentales con los compuestos químicos del grupo de órgano-fosforados, carbamatos y piretroides. Dichos accidentes se presentan en diversos momentos desde la compra y transporte, la aspersión sin elementos de protección personal (EPP) apropiados, hasta la forma de guarda y custodia de los equipos y ropa de trabajo, la cual de manera habitual involucra 
exposiciones incluso en el ámbito doméstico, pues los equipos (bombas de espalda) se aseguran en las habitaciones debajo de las camas y la ropa de trabajo es utilizada de manera continua varios días antes del lavado de las mismas. $(15,16)$

El equipo investigador en estudios descriptivos previos logró identificar en esta población de fumigadores signos y síntomas sugestivos de intoxicación crónica por exposición a compuestos químicos de uso agropecuario. Situación que motivó la realización del estudio que se presenta con el objetivo de asociar esta sintomatología sugestiva de intoxicación crónica con pruebas de laboratorio; con lo cual pretende identificar las reales condiciones de salud de esta población expuesta, con el fin de aportar soluciones viables a la problemática y prevenirla a futuro. (18)

\section{Materiales y métodos}

El área geográfica donde se localizaron los fumigadores participantes fue la zona rural de la región de sabanas de Córdoba (Colombia), conformada por siete municipios reconocidos por su vocación agrícola y pecuaria.

Se realizó un estudio analítico, transversal y prospectivo de dos años de duración, en el cual se aplicó el muestreo intencional mediante actividades sucesivas y complementarias en dos etapas a saber:

Primera etapa. Educación para el autocuidado y tamizaje. En sesiones educacionales para fomento del autocuidado de la salud en ambientes laborales, se capacitó al grupo de fumigadores asistentes en cuanto a uso de EPP, niveles de toxicidad de los compuestos que es- parce y medidas preventivas para la salud de las personas. Se realizaron las actividades para tamizaje y previo descarte de la muestra de aquellos individuos fumigadores que presentaban condiciones patológicas, consumo habitual de cafeína y nicotina; así como también a aquellos que reportaron el uso de medicamentos inhibidores de CE incluidos en la lista como Sulfonamidas y Lorazepam. En total la exclusión correspondió a $12 \%$ de los evaluados.

Segunda etapa. Consentimiento informado y toma de información. A cada uno de los 256 participantes seleccionados en la primera etapa se le ilustró debidamente sobre el proyecto, sus objetivos, alcances y métodos para obtener el consentimiento informado de su participación que involucraba la toma de cuatro muestras seriadas de sangre periférica con periodicidad semestral.

Ninguno de los sujetos participantes del grupo de estudio tenía resultados de laboratorio previo al inicio de la exposición, y que debido a que derivan su sustento familiar del trabajo diario era improbable obtener las cifras de referencia, pues implicaba someterlos a largos periodos de carencia (sin exposición); fue necesario seleccionar un grupo similar en población no expuesta a compuestos agroquímicos para obtener las cifras de referencia basales en esta población.

El mismo procedimiento de las etapas uno y dos se aplicó al grupo de no fumigadores formado por miembros de una comunidad étnica perteneciente a los cabildos indígenas residentes en las zonas de sabanas de Córdoba. Este grupo fue seleccionado debido a que en ocasión de su trabajo agropecuario no están expuestos a químicos agrícolas, pues su produc- 
tividad se realiza en condiciones de protección a la madre tierra.

Métodos. Mediante entrevista se tomaron datos de las características socio-demográficas, así como los antecedentes de exposición (edad de inicio, tiempo de trabajo, frecuencia diaria y semanal de exposición, compuestos químicos que utiliza, frecuencia de accidentalidad, compuestos involucrados en el accidente, tratamiento recibido); mediante una serie de visitas de campo a las áreas de labor se observaron las formas en que ocurren los incidentes de contacto accidental con pequeñas dosis del químico y los mecanismos de disposición final de residuos y empaques contenedores de los compuestos.

Así mismo se realizó valoración médica ocupacional en cuatro oportunidades (una cada seis meses). La primera, previa al conocimiento de los resultados de laboratorio, en la cual se buscaba de manera activa la identificación en cada sujeto de sintomatología asociada; y la segunda, con resultados de los exámenes. En esta sesión el médico y enfermeras ocupacionales brindaron educación específica al participante sobre sus riesgos individuales, sobre su estado de salud y se dieron recomendaciones para el trabajo y el hogar de manera que se controlaran los riesgos para la salud de los fumigadores y sus familias. Mediante cita previa prioritaria fueron remitidos los casos positivos identificados a la Empresa Promotora de Salud en la cual estaba afiliado el participante, con recomendaciones médicas para atención de la condición de salud identificada.

A cada trabajador agrícola seleccionado se le tomó, entre las 7:20 y las 11:20 de la mañana, una muestra de $10 \mathrm{ml}$ de sangre venosa peri- férica, la cual fue repartida en dos tubos con heparina que se colocaron en frío hasta llegar al laboratorio (4.2 horas aproximadamente). En el laboratorio, las muestras se conservaron refrigeradas a $2-8^{\circ} \mathrm{C}$ hasta el momento del procesamiento para su análisis (10 horas como máximo).

Las mediciones de CE se realizaron mediante la técnica $\mathrm{EQM}^{\circledast}$ en la cual para obtener los valores de colinesterasa eritrocitaria mediante espectrofotomería empleando el valor de la oxihemoglobina presente en la muestra de sangre; se consideraron como normales los valores de referencia para Colombia en población de hombres adultos no expuestos, siendo las cifras encontradas con la técnica $\mathrm{EQM}^{\oplus}$ correspondientes a $35,21 \mathrm{U} / \mathrm{g}$ de oxihemoglobina (IC95\%: 34,82U/mL a 35,60U/mL). Con la selección de los participantes, excluyendo otras causas de alteración de la colinestarasa, se pretendía controlar la posibilidad de falsos positivos y falsos negativos en la población estudiada. $(4,6,11)$

Para el análisis estadístico se usaron los programas Excel de Office para Windows 8, SPSS 9.0 y Epilnfo 6.04. En todas las pruebas estadísticas se aplicó un nivel de significación $\mathrm{p}$ de 0,05 .

La estadística aplicada se basó en medidas epidemiológicas y correlaciónales utilizando la corrección de significación de Lilliefors; los hallazgos se presentan en cuadros que agrupan la información de interés.

\section{Resultados}

Ninguno de los sujetos participantes del grupo de fumigadores, ni del grupo de no fumiga- 
dores se había medido la colinesterasa previo al inicio de la actividad laboral, ni durante el tiempo de ejercicio del trabajo como fumigador.

La totalidad de los participantes se encontraban y mantuvieron activos en su actividad laboral durante el periodo estudiado; estaban afiliados a régimen subsidiado de salud, sin vínculos con administradoras de riesgos laborales ni ninguna otra prestación social debido a la informalidad de su trabajo.

Las condiciones laborales para el 98\% de ellos involucra el trabajo independiente, con pago a destajo con ingresos diarios que no superan los 3 dólares (10.000 pesos colombianos - COP) percibidos por un jornal de 6 horas. La edad de inicio en la actividad laboral fue a los 16 años (73\%), el tiempo de exposición de 67\% de los sujetos del grupo de estudio corresponde a cifras entre 8 y 28 años de exposición a compuestos. $100 \%$ se mantuvo laboralmente activos durante los 24 meses del estudio y expuestos a la acción química de nueve grupos de compuestos tóxicos: órgano-fosforados, carbamatos, piretroides e inhibidores de quitina; Auxínicos o Fenoxi, derivados Picolínicos y Triazinas, amidas y ácidos alifáticos.

Los principales compuestos por tipo de uso utilizados son aquellos con efecto insecticida predominando los compuestos órgano-fosforados (74\%) entre los que se destacan los principios activos metil-parathión, clorpirifos, malatión, dimetoato, profenofos; seguido en orden de frecuencias por carbamatos (16\%), entre los que se encontró referencia a los ingredientes activos metomil, carbaril, thiodicarb; entre los piretroides (5\%) el compuesto cipermetrina; y entre los inhibidores de quitina (5\%) el compuesto activo más reportado fue el Lufenurón (ver cuadro 1).

Cuadro 1. Compuestos químicos insecticidas utilizados en la región de sabanas de Córdoba (Colombia).

\begin{tabular}{|l|l|}
\multicolumn{1}{|c|}{ GRUPO QUÍMICO } & \multicolumn{1}{c|}{ COMPUESTOS ACTIVOS } \\
& Methil Paration \\
\cline { 2 - 2 } Organo -fosforados (74\%) & Cloripirifos \\
\cline { 2 - 2 } & Profenofos \\
\cline { 2 - 2 } & Dimetoato \\
\cline { 2 - 2 } & Malation \\
\hline \multirow{4}{*}{ Carbamatos (16\%) } & Metomil \\
\cline { 2 - 2 } & Carbaril \\
\cline { 2 - 2 } & Tiocarb \\
\hline Piertroides (5\%) & Cipermetrina \\
\hline Inhibidores de quitina (5\%) & Leuferonon \\
\hline
\end{tabular}

Fuente. Primaria - sistematizado con el análisis de los reportes de uso en campo por José Moisés Luna Rondón. Universidad de Córdoba (Colombia).

Los participantes con frecuencia se exponen ocupacionalmente a cinco de estos nueve grupos químicos, $55.6 \%$ de los compuestos quí- micos tienen acción anticolinesterásica demostrada, y a ellos se exponen $98 \%$ de los sujetos valorados con precarias condiciones de protec- 
ción laboral. Pudo identificarse mediante observación en campo la exposición continuada de $92 \%$ de los sujetos en estudio a goteos, derrames y aspiración.

Las cifras de CE entre fumigadores y no fumigadores evidenció un comportamiento diferencial marcado, en el cual las cifras de los no fumigadores se mantuvo dentro del rango normal con ligeras variaciones dentro, pero con una tendencia sostenida de adherencia al límite inferior; en tanto los casos de fumigadores tuvieron un comportamiento oscilante con cifras iniciales por debajo de los valores de referencia, los valores obtenidos de colinesterasa eritrocitaria entre los fumigadores mostró fluctuaciones fuera del rango normal, interpretándose como la presencia de afectación o de inhibición. Estos descensos de la CE se evidenciaron con mayor claridad en el 67\% de los fumigadores en la tercera y cuarta medición (ver cuadro 2).

Cuadro 2. Comportamiento de la colinesterasa eritrocitaria entre fumigadores y no fumigadores.

\begin{tabular}{|c|c|c|c|c|}
\hline \multirow{3}{*}{ GRUPO } & \multicolumn{4}{|c|}{$\begin{array}{l}\text { Valores de referencia: } \\
34,82 \text { a } 35,60 \text { unidades por mililitro }(\mathrm{U} / \mathrm{mL}) \text {. } \\
\text { (Carmona- Fonseca, 2007) }\end{array}$} \\
\hline & \multicolumn{4}{|c|}{ Rangos de resultados } \\
\hline & $1^{\text {a }}$ medición & $2^{a}$ medición & $3^{\text {a }}$ medición & $4^{\mathrm{a}}$ medición \\
\hline Grupo fumigadores & $33,7-33,76$ & $32,9-33,73$ & $29,82-33,17$ & $27,4-32,76$ \\
\hline Grupo de no fumigadores & $34,2-34,96$ & $34,15-35,1$ & $34,16-35,2$ & $34,19-35,3$ \\
\hline Promedio fumigadores & 33,83 & 33,31 & 31,0 & 30,0 \\
\hline Promedio no fumigadores & 34,6 & 34,6 & 34,7 & 34,7 \\
\hline Sintomáticos (sugestivo IC) fumigadores & 30 & 35 & 38 & 37 \\
\hline Sintomáticos (sugestivos IC) no fumigadores & 2 & 3 & 1 & 3 \\
\hline
\end{tabular}

Fuente. Primaria: Conglomerado de los resultados de laboratorio con la técnica $\mathrm{EQM}^{\oplus}$

En relación con los promedios de CE entre fumigadores y no fumigadores evidencia el descenso progresivo entre los casos, lo cual contrasta con la relativa estabilidad entre los no fumigadores. El promedio de las 4 mediciones de CE oscila entre 33.8 y $27.6 \mathrm{U} / \mathrm{g}$ de oxihemoglobina, con descensos progresivos del valor inicial (67\%).

La distribución de sintomáticos de intoxicación crónica permitió identificar la presencia de entre 30 y 38 personas en esta condición entre fumigadores (9\%-11\%) entre los no fumigadores $(2,1 \%)$ se encontraron algunos sintomáticos, en particular las respuestas afectivas como depresión, desgano que pueden obedecer a otras causas endógenas o exógenas.

Además se pudo determinar que existe una aparente correlación positiva entre los resultados de la medición enzimática en las cuatro pruebas cuantitativas de actividad de la colinesterasa eritrocitaria con la presencia de síntomas de toxicidad crónica. 
En la valoración clínica se identificó la existencia de $37.2 \%$ de sintomáticos de intoxicación crónica por plaguicidas coincidentes con descensos sostenidos de la CE. En estos casos, los principales síntomas identificados mediante la valoración especializada entre los casos expuestos se relacionan con la sintomatología característica de sobreestimulación del sistema nervioso como contracturas musculares (74\%), hipertensión (62\%), cefalea (54\%); mareos-vértigos; irritabilidad, astenia, inquietud, debilidad y temblor se presentaron en $32 \%$ de los participantes; en tanto que los episodios de diarrea, diaforesis, sialorrea, visión borrosa, y miosis estuvieron presentes en
28\% de los casos; los síntomas de bradicardia e hipotermia y episodios de confusión entre los casos sintomáticos estuvieron cercanos al $8 \%$ de los sintomáticos.

La comparación realizada entre fumigadores de químicos agrícolas y no fumigadores (mo) quienes presentan condiciones de vida similares, excepto por la exposición continua a los agroquímicos plaguicidas como factor de riesgo $(m i)$ se realizó utilizando entre otras medidas estadísticas la razón de nomios o de posibilidades que es una medida de tamaño de efecto utilizando la tabla de 2x2 (ver cuadro 3 ).

Cuadro 3. Medición de la razón de nomios.

\begin{tabular}{|c|c|c|c|}
\hline & FUMIGADORES & INDÍGENAS & \\
\hline $\begin{array}{c}\text { Sintomáticos de } \\
\text { Intoxicación crónica }\end{array}$ & 140 & 9 & $\begin{array}{l}\text { Total de fumigadores } \\
\qquad \text { (mi) } 256\end{array}$ \\
\hline \multirow[t]{2}{*}{$\begin{array}{l}\text { Asintomáticos de } \\
\text { Intoxicación crónica }\end{array}$} & 116 & 247 & $\begin{array}{l}\text { Total de no fumigadores } \\
\qquad(m o) 256\end{array}$ \\
\hline & $\begin{array}{l}\text { Total positivos para } \\
\text { inhibición de la } \\
\text { colinesterasa } \\
\text { (ni) } 149\end{array}$ & $\begin{array}{l}\text { Total negativos para } \\
\text { inhibición de la } \\
\text { colinesterasa } \\
\text { (no) } 363\end{array}$ & $\begin{array}{l}\text { Población total } \\
\text { (n) } 512\end{array}$ \\
\hline
\end{tabular}

Fuente. Análisis estadístico de la información.

Los resultados de la razón de nomios se interpreta en sentido que cada fumigador tiene 33,1 veces más riesgo de presentar intoxicación crónica que un trabajador agrícola que en su trabajo no está expuesto de manera continua a inhibidores de colinesterasa. Se entiende que la presencia de sintomáticos para intoxicación crónica entre los controles podría ser debido a otras causas como depresión, ansiedad de leve a severa motivadas por causas externas transitorias que coincidieron con el periodo de muestreo.

Las pruebas estadísticas evidencian correlación entre los niveles de colinesterasa y la presencia de sintomáticos, el tiempo de exposición de 13 a 18 ańos y el uso inadecuado de los EPP, ver cuadro 4 . 
Cuadro 4. Correlación entre los niveles de colinesterasa y variables de interés.

\begin{tabular}{|c|c|c|c|c|c|c|}
\multicolumn{1}{|c|}{} & Síntomas & $\begin{array}{c}\text { Tiempo de } \\
\text { exposición (8- } \\
\mathbf{1 2} \text { ańos) }\end{array}$ & $\begin{array}{c}\text { Tiempo de } \\
\text { exposición } \\
\text { (13-18 ańos) }\end{array}$ & $\begin{array}{c}\text { Exposición } \\
\text { accidental } \\
\text { continuada }\end{array}$ & $\begin{array}{c}\text { Uso de EPP } \\
\text { inadecuados }\end{array}$ \\
\hline \multirow{2}{*}{$\begin{array}{c}\text { Niveles de } \\
\text { colinesterasa }\end{array}$} & $\begin{array}{c}\text { Coeficiente de } \\
\text { correlación }\end{array}$ & 0,949 & 0,463 & $0,948^{* *}$ & $-0,140 \quad 0,313^{* *}$ & 0,066 \\
\cline { 2 - 7 } & Sig. (bilateral) & 0,003 & 0,006 & 0,004 & 256 & 248 \\
\hline
\end{tabular}

Fuente. Análisis estadístico de la información.

\section{Discusión}

En el estudio se confirma mediante clínica y laboratorio la presencia de intoxicación crónica de origen ocupacional por exposición continuada a bajas dosis del compuesto químico entre un tercio de los participantes. Estos casos positivos para intoxicación crónica no ingresan, por tanto, en el Sistema de Vigilancia epidemiológica por su ausencia en los registros de la notificación semanal obligatoria a las unidades que hacen parte de la Red Nacional de Vigilancia de Colombia, evidenciando el subregistro de la problemática de salud laboral que aqueja a los trabajadores del sector informal (19-21).

Se identificaron hallazgos similares con Abou-Hatab y colaboradores, también con Henao y Corey quienes comprobaron que la actividad de la colinesterasa eritrocitaria no varía con la edad tal y como se comportó en esta población analizada con edades en el rango de 16 a 78 años $(17,22)$.

Si bien es cierto que la asociación entre la inhibición de la CE y la intoxicación crónica es un hecho demostrado hace muchos años, es la primera vez que se realiza un estudio en Córdoba (Colombia) orientado a determinar mediante resultados clínicos y de laboratorio la real condición de salud de los trabajadores informales dedicados a la fumigación en la actividad agropecuaria.

La utilidad de los resultados en la práctica de salud pública se da en aspectos educacionales a los sujetos de estudio, pero implica además la necesidad de formar a los profesionales de salud en la sospecha clínica e identificación del cuadro de intoxicación crónica y sus síntomas neurotóxicos, pues algunos autores han reportado que los sujetos sintomáticos de intoxicación crónica son medicados con vitaminas y suplementos para intentar controlar la astenia que acompaña el cuadro.

Se evidencia cómo la exposición continuada a plaguicidas se refleja en alteraciones de la $\mathrm{CE}$ entre los fumigadores informales, evidenciando intoxicación crónica con resultados de laboratorio que evidencian depresión de la actividad colinesterasica eritrocitaria y la presencia de manifestaciones clínicas prodrómicas. 
El comportamiento de la CE entre los fumigadores en comparación con los no fumigadores permite estimar una razón de nomios de 33,1 que indica más riesgo de alteración de la colinesterasa por agroquímicos-plaguicidas entre los fumigadores en comparación con los no fumigadores.

La sintomatología de la intoxicación crónica por plaguicidas puede generar factor de confusión con otra morbilidad, por lo que los cuadros clínicos pueden pasar inadvertidos hasta que esté muy avanzado el nivel de neurotoxicidad.

Conflicto de intereses. Los autores hacemos constar que no existe conflicto de intereses entre los autores y ninguna entidad pública o privada relacionada con la producción o comercialización de agroquímicos plaguicidas.

Entidad Financiadora del estudio. La investigación de donde se originó la publicación fue financiada por la Universidad de Córdoba (Colombia) con los recursos del Fondo de Investigaciones de la entidad.

\section{Referencias}

1. Colombia. Grupo de vigilancia y control de factores de riesgo ambiental. Protocolo de vigilancia y control de intoxicaciones por plaguicidas. 2010. En: https:// www.google.com.co/search?q=Protocolo + de +vigilancia $+y+$ control+de+intoxicaciones+por+plaguicidas\&rlz=1C1NHXL_esCO735CO736\&oq=Protocolo+de+vigilancia $+y+$ control+de+intoxicaciones+por+plaguicidas $\&$ aqs $=$ chrome..69i $57.2058 j$ 0 j8\&sourceid $=$ chrome $\& \mathrm{ie}=\mathrm{UTF}-8$

2. Idrovo, A.J. Vigilancia de las intoxicaciones con plaguicidas en Colombia. 2000. Revista de Salud Públi- ca, 2(1), 36-46.

3. Silva E, Morales L, Ortiz JE. Evaluación epidemiológica de plaguicidas inhibidores de acetilcolinesterasas en Colombia, 1996-1997. Biomédica. 2000;20:2009.

4. Carmona-Fonseca, J., Henao, S., y Garcés, R. Valores de referencia de actividad colinesterásica sanguínea en población laboral activa no expuesta a plaguicidas inhibidores de colinesterasa. (2000). Revista Facultad Nacional de Salud Pública, 18(2):55-72

5. Fernández, D.G., Mancipe, L.C., y Fernández, D.C. Intoxicación por organofosforados. (2010). Revista Med, 18(1), 84-92.

6. Carmona-Fonseca J. Colinesterasas en sangre total medidas con técnica semicuantitativa y en eritrocitos o plasma, medidas con técnicas cuantitativas: relaciones. Biomédica. 2007;27: 244-56

7. Díaz, I.C.B., Mosquera, N.L., Maquilón, J.K.P., y Hernández, J.J.A. Síndrome intermedio en intoxicación aguda por organofosforados: reporte de caso. Medicina UPB, (2012). 31(1), 53-58.

8. Arroyave, G., Feldman, R., Scrimshaw Y, y N.S. Estudio de los niveles séricos de colinesterasa en niños centroamericanos y su relación con el estado nutricional. (1962). Publicaciones científicas del Instituto de Nutrición de Centro América y Panamá, 6(59), 217.

9. Carmona-Fonseca, J. Colinesterasas eritrocitaria y plasmática en trabajadores con enfermedades crónicas controladas y en usuarios de medicamentos. (2006). Iatreia, 19(1):65-80.

10. Carmona-Fonseca J. Correlación y conversión entre los valores de actividad de la colinesterasa plasmática medida por tres técnicas diferentes. Rev Panam Salud Pública. 2007;22(1):35-40.

11. Carmona-Fonseca, J. Valores de referencia de la actividad de la colinesterasa eritrocitaria según las técnicas de Michel y EQM ${ }^{\bullet}$ en población laboral de Antioquia, Colombia. Rev Panam Salud Publica 2003.; 14(5): $316-324$ 
12. Marrugo, J., Lans, E., y Benítez, L. Hallazgo de mercurio en peces de la Ciénaga de Ayapel, Córdoba, Colombia. Revista MVZ Córdoba 2007;12(1), 878-886.

13. Nava, M.E.P. Aplicación de un instrumento para evaluar exposición a plaguicidas organofosforados, efectos agudos y subagudos en la salud de trabajadores agrícolas. Rev Fac Med UNAM, 2003; 46(1):22-27.

14. Teran, C.A.G., Dangond, H.V., y Rangel, A.C. Análisis epidemiológico y clínico de intoxicaciones agudas atendidas en Montería, Colombia. Revista Médica de Risaralda, 2015; 21(1):17-21.

15. Burger, M., y Fernández, S. Exposición al herbicida glifosato: aspectos clínicos toxicológicos. (2004). Revista Médica del Uruguay, 20(3), 202-207.

16. Puello-Alcocer, E.C., Ortega-Montes, J.E., y Valencia-Jiménez, N.N. Factores sociales asociados a la salud de los trabajadores informales agrícolas de la ciudad de Montería-Córdoba, Colombia. Revista Universidad y Salud. 2016; 15(2):103-112.

17. Abou-Hatab K, O’Mahony MS, Patel S, Woodhouse K. Relationship between age and plasma esterases. Age Ageing 2001;30(1):41- 45 .

18. Puello, EC y Luna, J. Morbilidad sentida y accidentalidad laboral por exposición a factores de riesgo químico en fumigadores de cultivos modales. 2007. Córdoba. Memorias I congreso Internacional y del Caribe en salud ambiental y ocupacional: Salud, Trabajo y Ambiente, Barranquilla, marzo de 2011, p110.

19. Zuluaga, M., Robledo, S., Osorio-Zuluaga, G., Yathe, L., Gonzalez, D., Taborda, G. Metabolómica y Pesticidas: Revisión sistemática de literatura usando teoría de grafos para el análisis de referencias. NOVA. 2016; 14 (26): 121-138

20. Romero, C., Castañeda, D., Acosta, G. Determinación de la calidad bacteriológica del aire en un laboratorio de microbiología en la Universidad Distri- tal Francisco José de Caldas en Bogotá, Colombia. NOVA. 2016; 14 (26): 129-137

21. Sierra, D., Bedoya, E. Prevalencia de hipoacusia neurosensorial inducida por ruido en empresas del sector madera de la ciudad de Cartagena. 2015. NOVA. 2016; 14 (26): 47-56

22. Henao S, Corey G. Plaguicidas inhibidores de las colinesterasas. Serie de Vigilancia 11. Metepec, México: Asistencia Editorial de Clemente Aguilar; 1991. p. 17-169. 